\title{
Progressive nicotine poisoning by multiple transdermal nicotine patches
}

\author{
Jeong Ho Kang ${ }^{1}$, Sung Kgun Lee 2 , \\ ${ }^{1}$ Department of Emergency Medicine, Jeju National University College of Medicine, Jeju, Republic of Korea \\ ${ }^{2}$ Department of Emergency Medicine, Jeju National University Hospital, Jeju, Republic of Korea
}

\begin{abstract}
The pharmacokinetic properties of transdermal nicotine patches (TNPs) are different from those of other routes of nicotine administration; further, acute nicotine poisoning by TNPs may present with different clinical features. In the present report, we describe the case of a 23-year-old woman who was admitted to emergency department (ED) at Jeju National University Hospital with loss of consciousness. Five hours before the ED visit, she used multiple TNPs to attempt suicide. Initially, nausea and vomiting occurred, and the symptoms worsened over time. We immediately removed the TNPs, and the application sites were gently washed with sterile water. The patient's level of consciousness gradually improved, and she fully recovered an altered mental status 5 hours later. Her initial urinary cotinine level was $324 \mathrm{ng} / \mathrm{mL}$. Physicians should be aware that acute nicotine poisoning by TNPs can cause various toxic symptoms.
\end{abstract}

Key words: Nicotine, Transdermal patch, Suicide

\section{INTRODUCTION}

Transdermal nicotine patches (TNPs) are widely used as nicotine replacement therapies to aid in smoking cessation. In many countries, various commercial TNP products are readily available to the public as over-the-counter (OTC) drugs. ${ }^{1,2)}$ They are easier to use than other routes of nicotine administration, so both adults and adolescents can easily use them to attempt suicide. Intentional poisoning by multiple TNPs can cause fatal nicotine toxicity, even death. ${ }^{2-6)}$

Nicotine is primarily metabolized in the liver and excret-

Received: June 9, 2021; Revised: July 19, 2021; Accepted: August 12, 2021

$\checkmark$ Correspondence to : Sung Kgun Lee

Department of Emergency Medicine, Jeju National University Hospital,

15 Aran 13gil, Jeju 63241, Republic of Korea

Tel: 82-64-717-1904, Fax: 82-64-717-1168

E-mail: casa0140@gmail.com ed through the kidneys, and its plasma half-life is estimated to be less than 1-2 hours. The lethal dose of nicotine in adults is approximately $0.5-1.0 \mathrm{mg} / \mathrm{kg}$, corresponding to a total dose of 30-60 mg. ${ }^{6,7)}$ Nicotine binds to nicotine-acetylcholine receptors (nAChRs), and its effect depends on the dose: small doses of nicotine stimulate the nAChRs, while higher doses inhibit them. ${ }^{4,7)}$ Higher doses of nicotine can also cause depression of the central nervous system (CNS), peripheral autonomic ganglia, and motor end plates of the skeletal muscles. Respiratory failure due to respiratory muscle paralysis and inhibition of the respiratory center in the brain are the leading causes of death from acute nicotine poisoning. $^{5-7)}$

In cases of acute nicotine poisoning by oral ingestion or intravenous injection, life-threatening conditions can occur within a few minutes. ${ }^{89)}$ However, TNPs have different 
pharmacokinetic properties and deliver nicotine gradually with no rapid uptake, so nicotine poisoning by multiple TNPs may have different clinical features. ${ }^{1)}$ In the present report, we describe the case of a patient who developed progressive nicotine toxicity by using multiple TNPs in an attempted suicide.

\section{CASE REPORT}

A 23-year-old woman was admitted to emergency department (ED) at Jeju National University Hospital with loss of consciousness. Four hours before the ED visit, she had tried to jump off a passenger ship into the sea. However, the cabin crew stopped this suicide attempt, and she was guarded and observed in the onboard infirmary. Initially, she was anxious and overexcited; she then complained of nausea and vomited several times. Subsequently, her level of consciousness gradually decreased. No detailed medical history was obtained because the patient's caregiver was not present.

The Table 1 shows the patient's vital signs and mental status in the ED. At the time of the ED visit, the patient's blood pressure was 107/63 mmHg; pulse rate, 68 beats/min; respiratory rate, 19 breaths/min; body temperature, 35.8; and blood sugar level, $111 \mathrm{mg} / \mathrm{dL}$. Her Glasgow Coma Scale (GCS) score was E2V3M4, and electrocardiography showed normal sinus rhythm. Arterial blood gas analysis revealed a $\mathrm{pH}$ of $7.41, \mathrm{pO}_{2}$ of $110 \mathrm{mmHg}, \mathrm{pCO}_{2}$ of $33.2 \mathrm{mmHg}$, and $\mathrm{HCO}_{3}$ levels of $22.0 \mathrm{mmol} / \mathrm{L}$. On physical examination, there were no other specific findings, but a total of eight TNPs were seen applied to the upper chest wall and both shoulders of the patient. We checked her belongings and found two TNP products: NICO-A ${ }^{\circledR}$ (SINIL Co. Ltd, Seoul, Korea) and NICOSTOP ${ }^{\circledR}$ (Samyang Biopharm Co., Seongnam, Korea). The dosages of the NICO-A ${ }^{\circledR}$ (SINIL Co. Ltd) and NICOSTOP ${ }^{\circledR}$ (Samyang Biopharm Co.) patches were $25 \mathrm{mg} / 16$ hours and $42 \mathrm{mg} / 24$ hours, respectively. The patient used four patches of each product.

We promptly removed the TNPs from the patient's body, and the application sites were gently washed with sterile water for 10 minutes or more. Urine samples were sent to a commercial laboratory to measure urinary cotinine levels; other laboratory tests showed no specific abnormalities. Conservative treatment and closed monitoring were per- formed. The patient's vital signs remained stable, and her GCS score gradually improved. Five hours after the ED visit, the patient had fully recovered, and we were able to perform additional history-taking. She was a non-smoker and had no specific underlying disease. She reported that she had experienced depressed mood for a month prior to the attempt, and her suicidal ideation had intensified from a week prior. She found information about the toxicity of nicotine through the Internet and purchased TNPs as OTC drugs at a pharmacy. Five hours before the ED visit, she had used the TNPs in a suicide attempt without any other toxic substances. We recommended that the patient be hospitalized for an additional observation period and for further psychiatric evaluation. However, she preferred to receive further treatment at her place of residence and was discharged. The results of the urinary cotinine test were available 5 days after patient discharge; levels were elevated to $324 \mathrm{ng} / \mathrm{mL}$ (reference: $<20 \mathrm{ng} / \mathrm{mL}$ ). The exemption of patient consent has been approved by the Institutional Review Board (IRB) Jeju National University Hospital (IRB No. 2020-11-004). All authors declare that this manuscript does not expose any personally identifiable information of the patient, and that this study poses no risk to the patient.

\section{DISCUSSION}

There are various routes of administration of nicotine, such as buccal (chewing gum, lozenges), topical (TNPs), intranasal (nasal spray pump), and oral inhalation (oral inhaler, e-cigarette). TNPs deliver nicotine at a more constant rate than other routes of administration, so the plasma nicotine concentrations increase gradually in proportion with

Table 1. Vital signs and mental status of the patient seen in the emergency department

\begin{tabular}{lccccc} 
Time (h) & $\begin{array}{c}\text { BP } \\
(\mathbf{m m H g})\end{array}$ & HR/min & RR/min & $\mathbf{S p O}_{2}(\%)$ & GCS \\
\hline 0 & $107 / 63$ & 68 & 22 & 94 & E2V3M4 \\
0.5 & $108 / 69$ & 63 & 20 & 100 & E2V2M6 \\
1 & $102 / 54$ & 56 & 20 & 100 & E3V5M6 \\
\hline 2 & $125 / 71$ & 54 & 20 & 100 & E4V5M6 \\
\hline 3 & $103 / 65$ & 58 & 20 & 100 & E4V5M6 \\
\hline
\end{tabular}

BP: blood pressure, HR: heart rate, RR: respiratory rate, GCS: Glasgow Coma Scale. 
the dose and time of application, with peak concentrations occurring 3-12 hours after TNP application, and the elimination half-life ranges from 3 hours to 6 hours after TNP removal. Nicotine absorption continues for several hours, even after removal, because the skin acts as a reservoir of nicotine. The bioavailability of nicotine from TNPs is approximately $80 \%$, probably because nicotine is lost from the edges of the patch. Nevertheless, there are significant differences in pharmacokinetics among commercially available TNPs. ${ }^{10,11)}$

Acute nicotine poisoning by multiple TNPs may have different clinical manifestations than nicotine overdoses by other routes. Buccal route-associated side effects include hiccups, irritation or tingling of the mouth and throat, oral mucosal ulceration, jaw-muscle ache, burping, unpleasant taste, dizziness, headache, and insomnia. Intranasal route-associated side effects include running nose, nasopharyngeal and ocular irritation, watery eyes, sneezing, and coughing. Oral inhalation-associated side effects include dyspepsia, oropharyngeal irritation, rhinitis, and headache. ${ }^{12)}$ Toxic symptoms may gradually progress from mild to severe, depending on the duration of TNP exposure. In the early phase, when the absorbed nicotine levels are low, patients may develop cholinergic symptoms such as headache, palpitations, dizziness, sweating, vomiting, and diarrhea. However, as the duration of TNP exposure increases, an inhibitory effect on nAChRs develops, which can cause life-threatening manifestations such as loss of consciousness, convulsions, respiratory muscle paralysis, fatal arrhythmia, and hypotension. ${ }^{2,5-7)}$ In the present case, approximately 52-66 mg of nicotine was gradually absorbed for $5 \mathrm{~h}$. As a result, the patient's clinical manifestations progressed from cholinergic symptoms to CNS depression symptoms after prolonged exposure to TNPs.

Acute nicotine poisoning by TNPs may be subject to delayed diagnosis, especially by inexperienced clinicians, because the initial symptoms are non-specific. To diagnose nicotine poisoning via TNP, an applied patch must be detected through physical examination or reported during history-taking. In addition, physicians must identify the commercial brand of TNP and the time of skin contact - there are differences in nicotine absorption rate and bioavailability among TNP products, and nicotine can be absorbed ex- tensively when a TNP has been applied for more than a few hours. ${ }^{3,5,6)}$ Toxicological assays of nicotine or cotinine levels are specific diagnostic tests for nicotine poisoning, but they may not be useful for initial diagnosis and treatment. Most samples are taken many hours after exposure, so the test results may not be correlated with symptom severity. Moreover, such assays may not always be possible, depending on the circumstances of the $\mathrm{ED}$, and if requested in a commercial laboratory, the results may take several days. ${ }^{2,13)}$

In patients with TNP poisoning, the patch must be removed as soon as possible, and the application sites should be washed with plain water, as soap can further increase the absorption of nicotine from the skin. $\left.{ }^{6}\right)$ Nicotine absorption can persist for several hours, even after a TNP is removed, so physicians should closely monitor the patient for at least 6 hours. If life-threatening symptoms are present, intensive treatments, such as mechanical ventilation and circulatory support, should be provided immediately. In the case of severe cholinergic symptoms or fatal bradycardia, atropine is recommended until symptoms improve. ${ }^{2,6,13)}$ In the present case, we detected and removed multiple TNPs early following detailed physical examination, and the application sites were gently washed with sterile water. These treatments blocked the further absorption of nicotine and prevented the exacerbation of toxic symptoms. We ultimately diagnosed acute nicotine poisoning based on the patient's elevated urinary cotinine levels; however, it took 5 days to confirm this result.

\section{CONCLUSIONS}

Although TNPs are easily purchased and used by the public, they can cause nicotine poisoning. Physicians should be aware that TNPs have pharmacokinetic properties different from those of other routes of nicotine administration. In patients with TNP poisoning, clinical manifestations may progress from mild to severe over time, and nicotine absorption persists for several hours, even after the TNP is removed. To diagnose and treat nicotine poisoning due to TNPs, patches must be detected early. Rapid removal of the TNP and washing of the application site prevent the worsening of toxic symptoms by blocking further absorption of nicotine. 


\section{REFERENCES}

1. Henningfield JE. Nicotine medications for smoking cessation. $\mathrm{N}$ Engl J Med 1995;333:1196-203.

2. Woolf A, Burkhart K, Caraccio T, Litovitz T. Self-poisoning among adults using multiple transdermal nicotine patches. J Toxicol Clin Toxicol 1996;34:691-8.

3. Kemp PM, Sneed GS, George CE, Distefano RF. Postmortem distribution of nicotine and cotinine from a case involving the simultaneous administration of multiple nicotine transdermal systems. J Anal Toxicol 1997;21:310-3.

4. Solarino B, Riesselmann B, Buschmann CT, Tsokos M. Multidrug poisoning involving nicotine and tramadol. Forensic Sci Int 2010;194:e17-9.

5. Tjoncke JA, Goncalves R, Castaing N, Molimard M, Tovagliaro F, Titier K. Death related to nicotine replacement therapy: a case report. Forensic Sci Int 2020;309:110223.

6. Montalto N, Brackett CC, Sobol T. Use of transdermal nicotine systems in a possible suicide attempt. J Am Board Fam Pract 1994; 7:417-420

7. Lardi C, Vogt S, Pollak S, Thierauf A. Complex suicide with homemade nicotine patches. Forensic Sci Int 2014;236:e14-8.
8. Kamble A, Khairkar P, Kalantri SP, Babhulkar S. Fatal suicidal attempt by deliberate ingestion of nicotine-containing solution in childhood-onset depression mediated through internet suicide guideline: a case report. Indian J Crit Care Med 2020;24:719-21.

9. Thornton SL, Oller L, Sawyer T. Fatal intravenous injection of electronic nicotine delivery system refilling solution. J Med Toxicol 2014;10:202-4.

10. Palmer KJ, Buckley MM, Faulds D. Transdermal nicotine. A review of its pharmacodynamic and pharmacokinetic properties, and therapeutic efficacy as an aid to smoking cessation. Drugs 1992;44:498529.

11. Fant RV, Henningfield JE, Shiffman S, Strahs KR, Reitberg DP. A pharmacokinetic crossover study to compare the absorption characteristics of three transdermal nicotine patches. Pharmacol Biochem Behav 2000;67:479-82.

12. Nicotine. [Internet]. Dallas, TX: Drugs.com; c2021 [cited 2021 Jul 29]. Available from: https://www.drugs.com/monograph/nicotine. html.

13. Sohn YD, Lee JS, Kang GH, Choi JT, Ahn ME, Seo JY, et al. Nicotine poisoning using nicotine patches. Journal of The Korean Society of Clinical Toxicology 2007;5:53-6. 